\title{
DISEÑO Y CONSTRUCCIÓN DE UN EQUIPO DE SOLDADURA POR FRICCIÓN CON ASISTENCIA LÁSER PARA LA UNIÓN DE EJES DE ACERO AISI 1045 Y ALUMINIO 2017-T4
}

\section{DESIGN AND CONSTRUCTION OF A FRICTION WELDING EQUIPMENT WITH LASER ASSISTANCE FOR THE JOINT OF AISI 1045 STEEL AND ALUMINUM 2017-T4 SHAFTS}

\author{
José Luis Mullo ${ }^{1,2}$ (D), Jorge Andrés Ramos-Grez ${ }^{1,3}$ (D), \\ Germán Omar Barrionuevo ${ }^{1, *}$
}

Recibido: 17-04-2021, Recibido tras revisión: 12-08-2021, Aceptado: 02-09-2021, Publicado: 01-01-2022

\section{Resumen}

La soldadura de materiales disímiles hace que los procesos de soldadura convencional no sean factibles de ser utilizados. La soldadura por fricción, por otro lado, ha demostrado ser una tecnología prometedora capaz de unir materiales sin que la temperatura supere su punto de fusión. Sin embargo, la obtención de las propiedades mecánicas de la junta soldada con características similares a los materiales base sigue siendo un desafío. En el desarrollo de este trabajo se diseñó y fabricó un equipo de soldadura por fricción rotatoria con asistencia láser. Para proporcionar el movimiento rotatorio se empleó un torno convencional de 3 HP de potencia y para generar fricción se diseñó un sistema hidráulico de presión el cual aplica fuerza axial mediante un cilindro de simple efecto para obtener la unión entre los materiales base.

\begin{abstract}
Welding metal alloys with dissimilar melting points make conventional welding processes unfeasible to be used. On the other hand, friction welding has proven to be a promising technology capable of joining materials, while preventing the temperature from exceeding the melting point. However, obtaining a welded joint with mechanical properties that are similar to the base materials remains a challenge. In the development of this work, a laser-assisted rotary friction welding equipment was designed and manufactured. A 3 HP conventional lathe was used to provide rotary movement, and a hydraulic pressure system that applies axial force through a simple effect cylinder was designed to generate friction to obtain the union between the base materials.
\end{abstract}

\footnotetext{
$\overline{1, *}$ Departamento de Ingeniería Mecánica y Metalúrgica, Escuela de Ingeniería, Pontificia Universidad Católica de Chile, Av. Vicuña Mackenna 4860, Macul, Santiago, Chile. Autor para correspondencia gobarrionuevo@uc.cl

${ }^{2}$ Carrera de Tecnología Superior en Mecánica Automotriz, Instituto Superior Tecnológico Ciudad de Valencia (ISTCV), Quevedo, Ecuador

${ }^{3}$ Centro de Investigación en Nanotecnología y Materiales Avanzados (CIEN-UC), Av. Vicuña Mackenna, 4860, Macul, Santiago, Chile. 
En el equipo implementado se realizaron uniones de ejes de acero AISI 1045 con aluminio 2017-T6; las uniones soldadas se evaluaron metalúrgicamente, haciendo hincapié en la composición química en la interfaz de la soldadura. Para la caracterización de la microestructura se empleó microscopía electrónica de barrido (SEM), espectroscopía de dispersión de energía (EDS) y espectrometría de emisión óptica de descarga luminiscente (GDOES) para medir la composición inicial de los materiales que se soldaron. Los resultados obtenidos muestran una adecuada unión entre los materiales base, denotando la utilidad del equipo fabricado para la unión de materiales disimiles.

Palabras clave: acero, aluminio, láser, materiales disímiles, microestructura, soldadura por fricción
In the implemented equipment, joints of AISI 1045 steel and 2017-T6 aluminum shafts were made. The welded joints were metallurgically evaluated, emphasizing on the chemical composition at the weld interface. For microstructure characterization, scanning electron microscopy (SEM), energy dispersion spectroscopy (EDS), and glow discharge optical emission spectrometry (GDOES) were used to measure the initial composition of the welded materials. The results obtained show an adequate joint between the base materials, denoting the usefulness of the equipment manufactured for the union of dissimilar materials.

Keywords: Steel, Aluminum, Laser, Dissimilar materials, Microstructure, Friction welding 


\section{Introducción}

Los procesos de unión de metales se constituyen en una necesidad esencial para diversas industrias relacionadas con la metalurgia y soldadura. Dependiendo del sector, la adecuada selección de materiales y procedimientos, junto con las normas de seguridad y calidad, son aspectos esenciales en la industria ingenieril [1]. En el instante que se requiere de la unión de materiales con características distintas, la soldadura convencional por fusión no es factible debido a sus limitaciones metalúrgicas. En la actualidad, los procesos convencionales no son suficientes debido a la diferencia entre sus puntos de fusión que generan compuestos intermetálicos de características frágiles y nada beneficiosos a la junta soldada [2]. En función de esta problemática, la soldadura de estado sólido ha sido un proceso de unión prometedor; en el cual, dos piezas de trabajo se unen bajo presión, generando calor por fricción a temperaturas inferiores al punto de fusión de los materiales base [3]. Dicha soldadura por fricción (FW) ha sido un método alternativo de unión por estado sólido, puesto que produce una coalescencia de materiales bajo una fuerza de compresión entre dos piezas de trabajo que giran o se mueven en contacto produciendo calor y desplazando plásticamente el material hasta crear una interfaz de contacto [4]. El metal de aporte, el fundente y el gas de protección no son necesarios en este proceso, debido a su simplicidad. El proceso enunciado sobre la base de estudios académicos, lo ha ubicado entre uno de los mejores para la producción en serie de unión en materiales disímiles [5]. Por esta razón, el proceso FW es utilizado en la industria automotriz, aeronáutica, petrolera, eléctrica, entre otras.

Debido a que las uniones de aceros con otros materiales en los procesos de soldadura por fricción pueden tener propagaciones de fases inesperadas, corrosión del límite de grano o generación de fases de ferrita delta y sigma en la interfaz de soldadura. Se ha considerado ciertas precauciones, como determinar las variables respecto a tratamientos térmicos, incrementar la velocidad de soldadura, ya que de esta manera se logrará una cierta homogeneidad en la distribución de temperatura en los dos componentes [6]. Bajo este contexto, la obtención de uniones soldadas con adecuadas propiedades mecánicas y metalúrgicas aportaría al interés creciente en una amplia gama de aplicaciones industriales. La fabricación de componentes en la industria del transporte y aeronáutica, requieren de elementos cilíndricos soldados con buenas propiedades mecánicas, bajo peso específico y buena resistencia a la corrosión. Concretamente, la introducción de piezas de acero y aluminio en sistemas rotatorios y en estructuras de acero requieren del desarrollo de procesos de unión fiables, eficientes y económicos. A través de la aplicación de un haz láser se podría optimizar la calidad de soldadura en un proceso FW convencional. Li, Yu, Li, Zhang y Wang [7] mencionan que, durante el proceso FW convencional, la generación de calor está determinada principalmente por la velocidad de rotación, la presión de fricción y el tiempo de fricción. Así, la energía de calentamiento es muy limitada, especialmente para la soldadura de ejes delgados.

La junta de soldadura del proceso de soldadura por fricción rotatoria es la parte más importante y que se debe analizar, misma que se caracteriza por una estrecha zona afectada por el calor ZAC, la presencia de material plásticamente deformado alrededor de la soldadura (flash) y la ausencia de una zona de fusión. Taban, Gould y Lippold [8] realizaron uniones entre ejes de una aleación de aluminio 6061-T6 y un acero AISI 1018, utilizando velocidades de rotación de hasta $4200 \mathrm{rpm}$, presión de fricción de $23 \mathrm{MPa}$, tiempo de fricción de $1 \mathrm{~s}$, tiempos de forja de $5 \mathrm{~s} \mathrm{y}$ presión de forja de $60 \mathrm{MPa}$. Los resultados mostraron una resistencia a la tracción de $250 \mathrm{MPa}$ con fallas en la zona plastificada debido a la existencia de una interfaz con compuesto intermetálico $\mathrm{Al}-\mathrm{Fe}$, con un espesor de $250 \mathrm{~nm}$ relacionado con las fases $\mathrm{Fe}_{2} \mathrm{Al}_{5}$ que se forman a temperaturas superiores a $1200{ }^{\circ} \mathrm{C}$ [9]. Handa y Chawla [10] adaptaron un torno convencional con un sistema de presión y frenado para crear una máquina de RFW. Los autores encontraron que la resistencia máxima a la tracción y la resistencia al impacto son aceptables a una velocidad de rotación de $1250 \mathrm{rpm}$ con una presión axial de $120 \mathrm{MPa}$, para juntas soldadas de acero AISI 1021 de $20 \mathrm{~mm}$ de diámetro. Luo et al. [11], por su parte, desarrollaron un método de unión entre un amortiguador y su vástago, con la finalidad de reducir las rebabas de soldadura. De esta manera, determinaron que, con una adecuada selección de los parámetros, el proceso de soldadura por fricción es muy eficaz para este tipo de aplicaciones, a diferencia de los métodos convencionales ya que la microestructura obtenida presenta granos más uniformes en ausencia de cambio de fase, lo que no sucede con los procesos de soldadura por fusión.

Las principales ventajas del proceso CDFW son el ahorro de material, el bajo tiempo de producción y la posibilidad de soldar materiales disímiles. En la actualidad se utiliza para la fabricación de elementos de la industria automotriz como válvulas, engranajes, semiejes, ejes del ventilador turbo, conexiones, pistones, etc. Para elaborar dichos elementos se emplean aleaciones ferrosas y no ferrosas [12]. Debido a su versatilidad, la soldadura por fricción tiene un uso industrial generalizado como un proceso de producción en masa para la unión de materiales [5]. Sin embargo, para realizar uniones disímiles, el proceso $\mathrm{FW}$ requiere de métodos alternativos de precalentamiento con la finalidad de brindar mejores propiedades en los materiales soldados. Por tal motivo, en el desarrollo del presente trabajo, se construyó un equipo de soldadura por fricción rotatoria donde, además, se aplica un pre- 
calentamiento mediante láser para acelerar el proceso y mejorar el acople entre los materiales base.

Para este experimento, el principio de funcionamiento se ilustra en la Figura 1, que describe las etapas del experimento mediante una asistencia láser en su etapa de diseño. En el desarrollo realizado, $\omega$ representa la velocidad de rotación en el extremo izquierdo, $F_{1}$ la fuerza de fricción y $F_{2}$ la fuerza de forja. Para esta configuración un haz de láser actúa sobre uno de los materiales, aumentando su temperatura antes de que comience el proceso de soldadura por fricción rotatoria $(\mathrm{RFW})$.

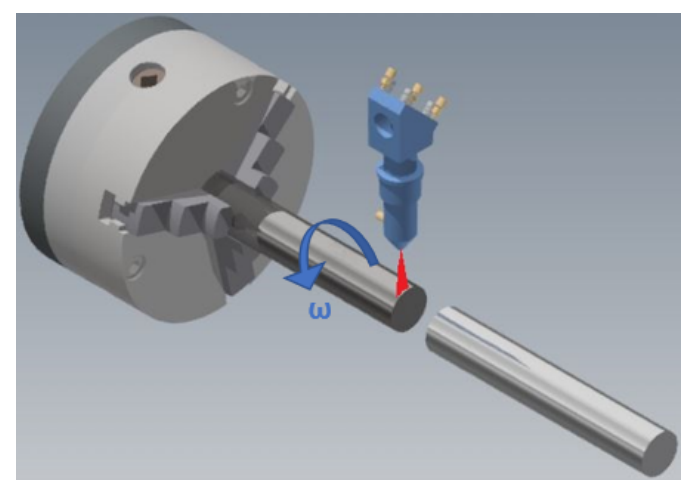

(a)

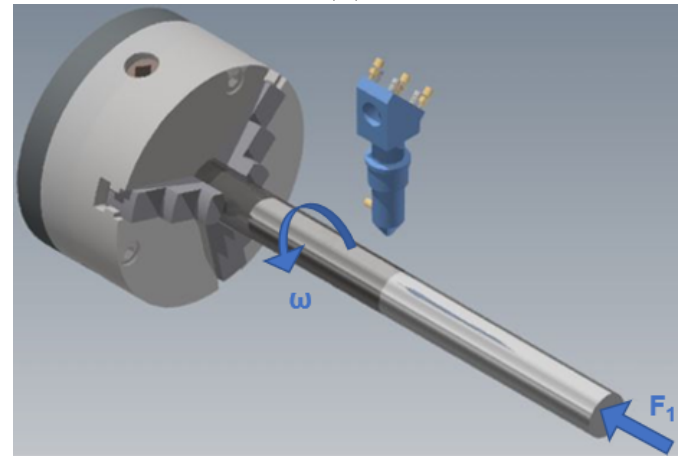

(b)

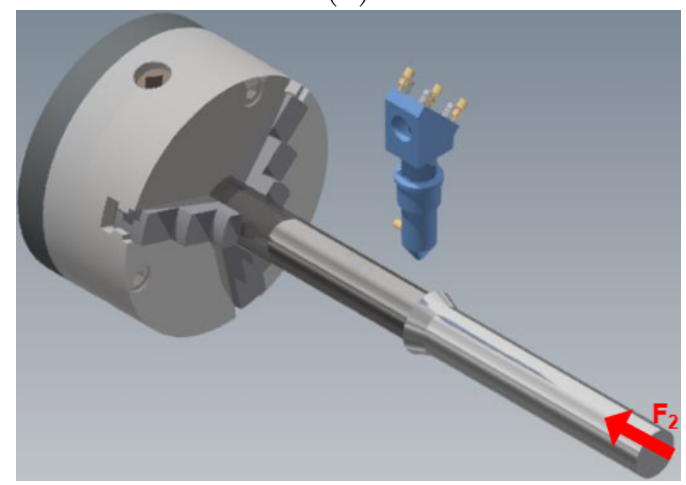

(c)

Figura 1. Etapas del proceso de soldadura por fricción con asistencia láser. a) Rotación del eje de acero y accionamiento del láser. b) Fin de la aplicación láser e inicio del proceso de fricción mediante la aplicación de la fuerza F1. c) Suspensión de la rotación y aplicación de la fuerza axial de forja [13]

\section{Materiales y métodos}

Para el proceso de diseño se realizó una profunda revisión de literatura donde se identificaron las publicaciones que presentan soldadura por fricción rotaria conocida como Continuous drive friction welding (soldadura de fricción por accionamiento directo) [14-19]. En este proceso se identifican dos mecanismos esenciales: uno de rotación y otro de presión. Para dar movimiento al eje se ha seleccionado un torno convencional de 3 HP de potencia, $2000 \mathrm{rpm}$ de velocidad de rotación y una distancia entre centros de $1000 \mathrm{~mm}$. Mientras que para el sistema fricción y forja se diseñó un sistema de presión operado hidráulicamente que permite realizar juntas de fricción a varios niveles de presión. El mecanismo de presión consta de un cilindro hidráulico de cinco toneladas de capacidad, impulsado por una bomba hidráulica de $300 \mathrm{cc}$. El mecanismo de presión también cuenta con un manómetro que permite visualizar las presiones de fricción y forja.

Para el diseño del mecanismo de presión (Figura 2) se acopló un contrapunto de presión al torno, el diseño se realizó en el software de diseño 3D Autodesk Inventor. Se utilizaron materiales de acuerdo con el grado de esfuerzo a soportar en el proceso de soldadura. La estructura del dispositivo está construida en acero AISI 1020 y para las partes que requieren un mayor esfuerzo como la boquilla de ajuste, el bloque amortiguador de vibraciones y la brida de sujeción se utilizaron aceros AISI 1045 y AISI 4120, respectivamente.

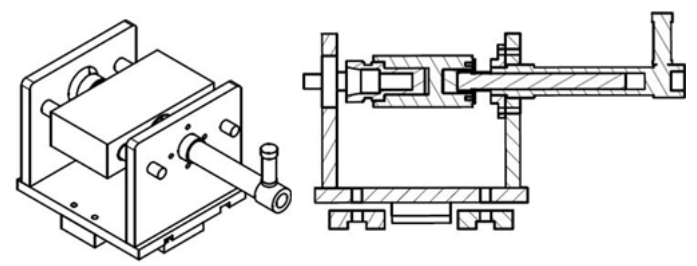

(a)

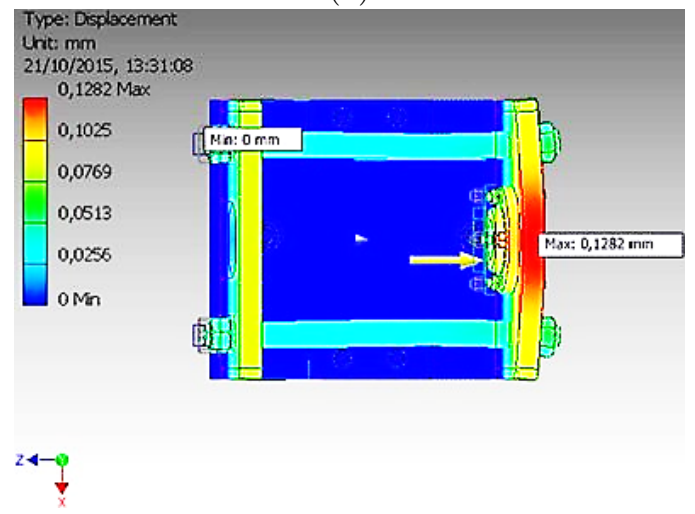

(b)

Figura 2. Diseño del sistema de sistema de presión hidráulico, a) Diseño asistido por computador, b) simulación del desplazamiento máximo 
La Figura 2a muestra el contrapunto diseñado. Utilizando ingeniería asistida por computador (CAE), las partes del sistema se sometieron a una fuerza de $1000 \mathrm{~kg}$ para verificar el esfuerzo máximo y su deformación en los ejes. En la Figura 2b se puede observar la máxima deformación sobre el eje $\mathrm{X}$, con un valor de $0,1282 \mathrm{~mm}$, lo cual es aceptable para que las uniones de soldadura sean concéntricas y de calidad.

Para el diseño hidráulico se consideró que la máxima fuerza entre probetas es de $1000 \mathrm{~kg}$ de acuerdo con la investigación de [20]. La fuerza establecida, permitirá realizar soldaduras en ejes de acero o aluminio con diámetros de hasta $20 \mathrm{~mm}$ de diámetro. Para determinar la presión hidráulica se realizó una prueba experimental utilizando la máquina universal de ensayos por compresión. Al aplicar presión el pistón hidráulico y medir la fuerza máxima alcanzada se obtiene un valor de presión que permite establecer las capacidades del sistema. Para cada valor de presión en el cilindro se presenta una Tabla 1 de análisis de las presiones.

Tabla 1. Presiones de prueba

\begin{tabular}{ccc}
\hline $\begin{array}{c}\text { Presión del } \\
\text { cilindro } \\
(\mathbf{M P a})\end{array}$ & $\begin{array}{c}\text { Fuerza } \\
\text { aplicada } \\
(\mathbf{k g})\end{array}$ & $\begin{array}{c}\text { Desplazamiento } \\
(\mathbf{m m})\end{array}$ \\
\hline 25,4 & 254 & 0,93 \\
52 & 520 & 1,27 \\
100 & 1000 & 1,79 \\
149,9 & 1499 & 2,21 \\
200,4 & 2004 & 2,62 \\
624,4 & 6244 & 5.17 \\
\hline
\end{tabular}

Como fuente de calor se empleó un equipo láser de $\mathrm{CO}_{2}$ (Oerlikon OPL3500) de $3,5 \mathrm{~kW}$ de potencia, que dispone de una longitud de onda de 10,6 $\mu \mathrm{m}$ y TEM00 (Figura 3). Mediante el cual se aplica un precalentamiento para que la unión entre los materiales base sea más eficiente de acuerdo con los resultados reportados en la literatura [21-27].

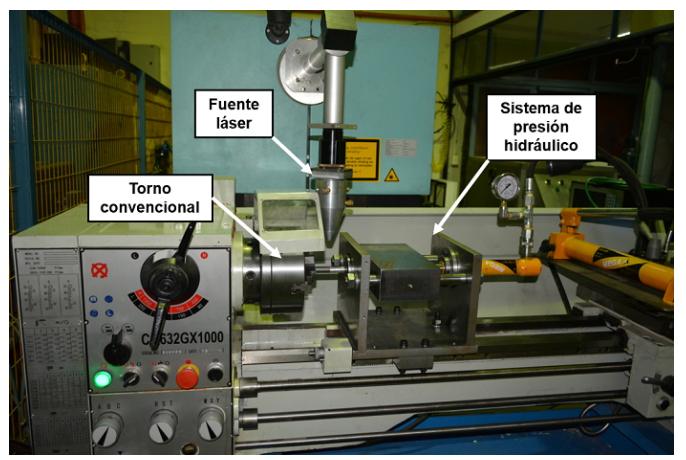

Figura 3. Equipo de soldadura por fricción con asistencia láser (LAFW)
El proceso de soldadura por fricción se realizó sobre ejes de acero AISI 1045 y ejes de aluminio 2017-T4 de $15 \mathrm{~mm}$ de diámetro y $180 \mathrm{~mm}$ de longitud cada uno. Las composiciones químicas nominales de los materiales base se informan en la Tabla 2. Las propiedades mecánicas de los materiales base se presentan en la Tabla 3.

Tabla 2. Composición química de los materiales base

\begin{tabular}{ccccc}
\hline Material & \multicolumn{4}{c}{ Elementos (wt \%) } \\
\hline \multirow{4}{*}{ AISI 1045 } & $\mathrm{Fe}$ & $\mathrm{C}$ & $\mathrm{Mn}$ & $\mathrm{Si}$ \\
& 98.41 & 0.40 & 0.72 & 0.22 \\
\cline { 2 - 5 } & $\mathrm{Cu}$ & $\mathrm{Al}$ & $\mathrm{P}$ & $\mathrm{S}$ \\
& 0.13 & 0.02 & 0.01 & 0.01 \\
\hline \multirow{4}{*}{$\mathrm{AA} 2017$} & $\mathrm{Al}$ & $\mathrm{Cu}$ & $\mathrm{Mg}$ & $\mathrm{Mn}$ \\
& 92.92 & 4.25 & 1.58 & 0.84 \\
\cline { 2 - 5 } & $\mathrm{Fe}$ & $\mathrm{Zn}$ & $\mathrm{Cr}$ & $\mathrm{Si}$ \\
& 0.34 & 0.04 & 0.01 & 0.002 \\
\hline
\end{tabular}

Tabla 3. Propiedades mecánicas de los materiales base

\begin{tabular}{cccc}
\hline Material & $\begin{array}{c}\text { Esfuerzo a } \\
\text { la rotura } \\
\text { (MPa) }\end{array}$ & $\begin{array}{c}\text { Esfuerzo a } \\
\text { la fluencia } \\
\text { (MPa) }\end{array}$ & $\begin{array}{c}\text { Dureza } \\
\text { (HV) }\end{array}$ \\
\hline AISI 1045 & $617-680$ & $330-392$ & $260-330$ \\
AA 2017 & $370-420$ & $215-260$ & $105-120$ \\
\hline
\end{tabular}

El proceso de unión consiste en precalentar el eje de acero durante 40 segundos; el proceso de fricción se inicia hasta que se alcanza la velocidad de rotación entre 1600 y $1800 \mathrm{rpm}$, luego se aplica la presión de fricción axial (14/21 MPa) mediante el mecanismo hidráulico hasta lograr la forja durante 60 segundos. Finalmente, se aplicó la presión de forjado (42,1 $\mathrm{MPa})$ durante 40 segundos. Estos valores de parámetros constantes se eligieron después de varios ensayos previos, en los que se encontró que eran los valores óptimos con respecto a la capacidad del hardware utilizado (Figura 4).

Precalentamiento (40)

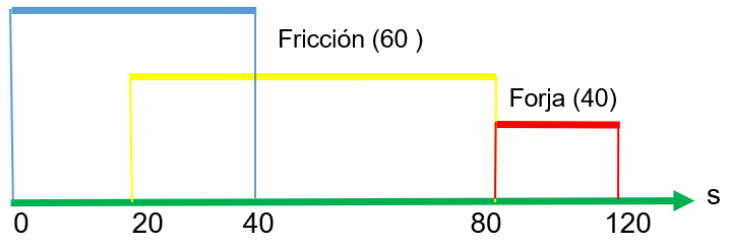

Figura 4. Parámetros del proceso de soldadura

Donde, para el tiempo de precalentamiento láser la presión, $\mathrm{P}=0 \mathrm{MPa}$. Posteriormente para el tiempo de fricción, la presión de fricción $\mathrm{Pf}=14$ y $21 \mathrm{MPa}$. Finalmente, en el tiempo de forja se incrementa la presión de forja a $\mathrm{Pu}=42,1 \mathrm{MPa}$. 
Para la evaluación macro y microestructural se analizaron 12 probetas obtenidas con el proceso de soldadura con asistencia láser. Las secciones transversales de las uniones soldadas fueron sometidas a una preparación metalográfica de pulido grueso con lijas de 200, 400, 600, 800 hasta la lija de grano 1000 acorde la norma ASTM E407. Se aplicó un pulido fino con alúmina de $0,04 \mu \mathrm{m}$ de diámetro promedio de grano y un abrasivo de pasta de diamante de 0,01 $\mu \mathrm{m}$ de diámetro para evitar relieves en la superficie de la muestra. El equipo que se utilizó para observar la microestructura es un microscopio marca MEIJI modelo IM 7200 con una amplificación de hasta 100x, 500x y $1000 \mathrm{x}$.

Con la finalidad de visualizar las fases presentes y el tamaño del grano se realizó el ataque químico en el acero mediante inmersión por 10 segundos en una solución de Nital (alcohol al $95 \%$ y ácido nítrico $\mathrm{HNO}_{3}$ en $5 \mathrm{ml}$ ). Para el componente aluminio se impregnó por 15 segundos un cotonete con una solución de ácido fluorhídrico (1 ml de fluoruro de hidrógeno $\mathrm{HF}$ y $200 \mathrm{ml}$ de $\mathrm{H}_{2} \mathrm{O}$ ). La medición de tamaño de grano se realizó bajo la norma ASTM E112 usando el método de intercepción para todos los casos. Finalmente, se realizó una comparativa entre el proceso FW convencional y el proceso LAFW desarrollado por los autores.

\section{Resultados y discusión}

Durante el proceso de soldadura, se observa la aplicación del láser al acero y posteriormente a la aplicación de la fricción y forja se obtiene la formación de un flash alrededor del eje de aluminio (Figura 5).

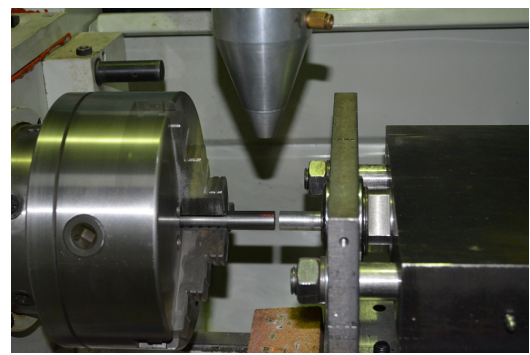

(a)

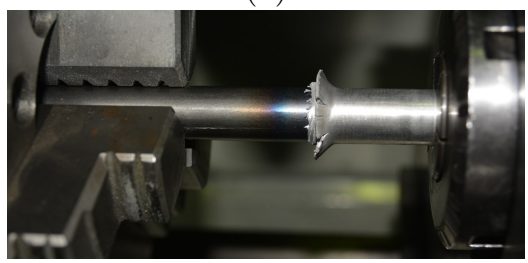

(b)

(c)

Figura 5. Proceso de soldadura. a) Aplicación láser sobre el eje de acero. b) Formación del flash en el eje de aluminio. c) Probeta soldada
La formación del flash está influenciada principalmente por las características del material sumadas a la presión de fricción, lo que lleva a una deformación más significativa, provocando un mayor efecto de flash debido a la mayor presión mecánica [20].

En general el aspecto de la unión de soldadura para las condiciones establecidas es simétrico, la presión de fricción influye principalmente en un efecto flash que, generalmente, ocurre en el lado del aluminio [8]. Una presión mecánica mayor conduce a una mayor deformación del material que a su vez provoca un mayor efecto de flash (Figura 6).

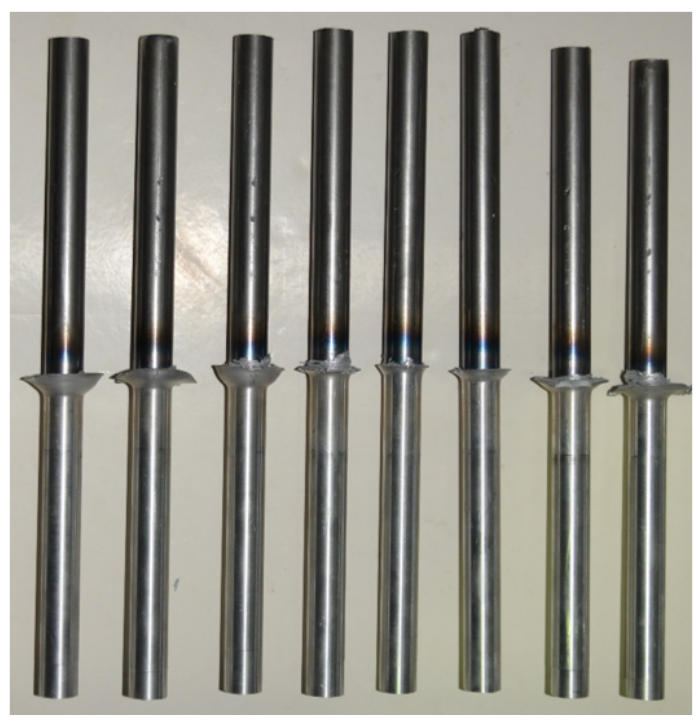

Figura 6. Probetas soldadas, donde se puede observar distintas configuraciones del flash sobre el eje de aluminio

La unión entre aluminio y el acero comienza desde la región exterior y se dirige hacia el centro. La unión mostrada en la Figura 7a presenta una unión homogénea, debido al aporte de calor adicional del láser que favorece a la homogenización del material permitiendo se fusionen ambos materiales [14]. Para determinar la calidad de la unión es necesario realizar el análisis de la microestructura realizando el ataque químico, para verificar la deformación del aluminio en el acero (Figura 7b).

Para uniones acero-aluminio producidas mediante soldadura por fricción se conoce que existen cuatro zonas definidas desde los extremos hacia el centro: zona no deformada (ZND), zona afectada térmicamente (ZAT), zona afectada termomecánicamente (ZATM) y la capa de reacción o interfaz Fe-Al. En el caso del acero, la ZND presenta una matriz conformada por ferrita y perlita en aproximadamente un 39,36 \% y un $60,64 \%$, respectivamente. La matriz tiene un porcentaje de carbono de un $0,45 \%$ y un tamaño de grano ASTM 6 que corresponden a un acero AISI 1045 (Figura 8a). La ZAT muestra un ligero refinamiento del grano similar a los obtenidos por [28]. La zona de 
microestructura más crítica es la ZATM que se caracteriza por un refinamiento mayor del grano, debido a que existe un incremento de la temperatura y rápido enfriamiento. Respecto al aluminio, la ZND presenta una matriz conformada por aluminio y cobre en aproximadamente un $93,85 \%$ y un $6,15 \%$, respectivamente. La matriz tiene un tamaño de grano ASTM 10 que corresponde a un aluminio AA 2017 (Figura 8b).
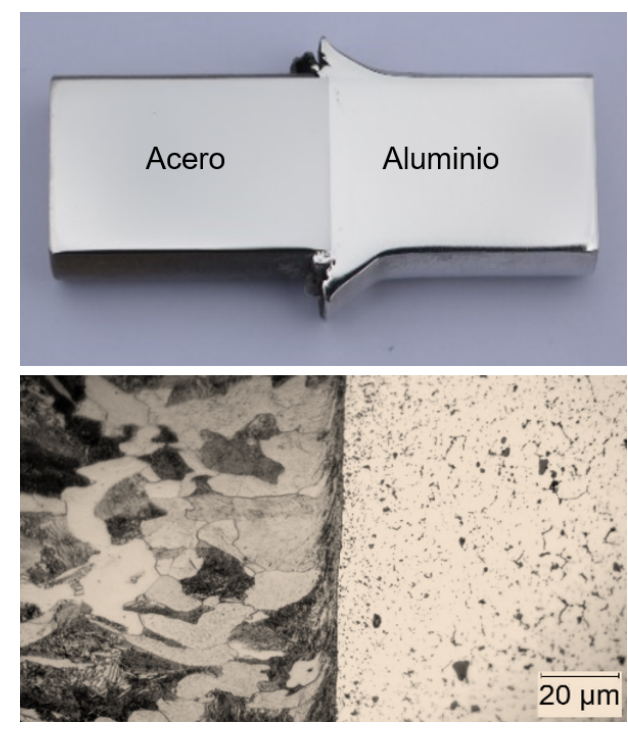

Figura 7. Sección transversal - Micrografía de la unión acero-aluminio 500x

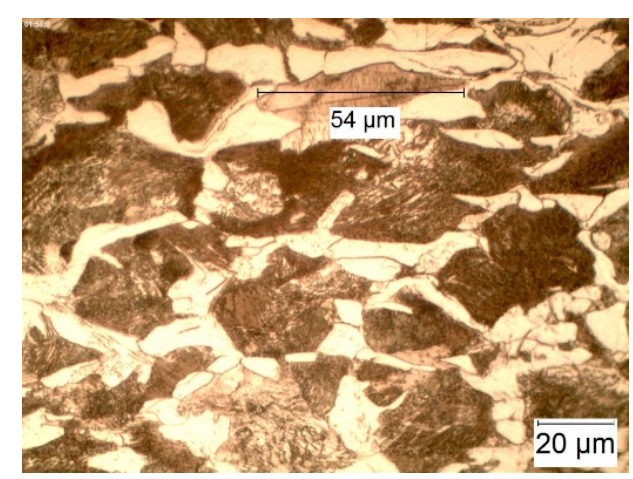

(a)

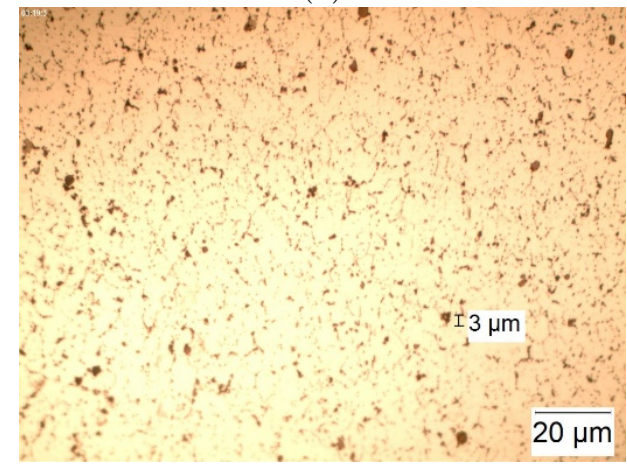

(b)

Figura 8. Microscopía óptica de la zona afectada termomecánicamente a 10000x a) Acero. b) Aluminio.
La ZAT presenta un refinamiento del grano similar a los obtenidos por [29]. En la (ZATM) existe una mayor deformación termomecánica producto de la fusión por fricción al tratarse de un material con un menor punto de fusión que el acero.

En referencia a la ZATM con asistencia láser se analizó a una amplificación de 1000x como lo describe la Figura 8a; en la Tabla 4 se puede observar el porcentaje de la matriz perlita a 53,51\%, además, aparece ferrita acicular en un 46,49\% producto del calentamiento y rápido enfriamiento producidos por la presión de fricción y la asistencia láser. El tamaño de grano es ASTM 6 con una dimensión promedio aproximada de 54 micrones calculado a través del método de interceptos. En la Figura 8b existe aluminio y cobre con un 94,56 y $5,44 \%$ respectivamente. Se tiene un tamaño de grano ASTM 9 y un diámetro máximo de tres micrones.

Tabla 4. Fases y elementos presentes en los materiales base

\begin{tabular}{ccc}
\hline \multicolumn{4}{c}{ AISI 1045 } \\
\hline Área de perlita & 1020967,40 & $\mu^{2}$ \\
Área de ferrita & 887088,60 & $\mu^{2}$ \\
Perlita \% & $53,51 \%$ & \\
Ferrita \% & $46,49 \%$ & \\
Área total & 1908056 & $\mu^{2}$ \\
\hline \multicolumn{4}{c}{ AA } & $\mathbf{2 0 1 7 - T 4}$ & \\
\hline Área de cobre & 103810,60 & $\mu^{2}$ \\
Área de aluminio & 1804245,40 & $\mu^{2}$ \\
Cobre \% & $5,44 \%$ & \\
Aluminio \% & $94,56 \%$ & \\
Área total & 1908056 & $\mu^{2}$ \\
\hline
\end{tabular}

La Figura 9 muestra los puntos de análisis para determinar la composición química de cada material mediante espectrometría de emisión óptica de descarga luminiscente (GDOES), aluminio a la izquierda y acero a la derecha. En el centro se tiene la interfaz de soldadura (punto 3). Los resultados obtenidos se presentan en la Tabla 5. Se distingue claramente la mayor concentración de aluminio (Al) alrededor del $82 \%$, cobre $(\mathrm{Cu})$ cerca de $9 \%$ y manganeso $(\mathrm{Mn})$ sobre $5 \%$ para la aleación 2017-T4. Para el acero 1045 se tiene una concentración de hierro (Fe) mayor a $97 \%$. Mientras que en la interfaz se redistribuye la composición química en Fe $62 \%$ y Al $33 \%$, con menor contenido de $\mathrm{Cu}$ y $\mathrm{Mn}$.

Para complementar el análisis de la microestructura, la Figura 10, muestra un mapeo elemental mediante espectroscopía de energía dispersiva (EDS). Se puede observar la variación del contenido de elementos a lo largo de la interfaz de soldadura. En la parte superior se presenta $\mathrm{Fe}$ a mayor concentración identificado por el color rojo correspondiente a la aleación de acero 
Mullo et al. / Diseño y construcción de un equipo de soldadura por fricción con asistencia láser para la unión de ejes de acero AISI 1045 y aluminio 2017-T4

1045. En la parte inferior se distingue el Al en color amarrillo correspondiente a la aleación 2017-T4. También es posible distinguir una elevada concentración de $\mathrm{Cu}$ en la parte inferior y en menor medida silicio (Si) tanto en la aleación de acero como en el aluminio.

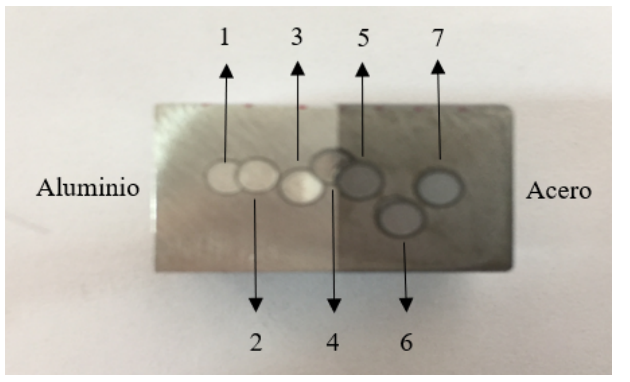

Figura 9. Espectrometría de emisión óptica de descarga luminiscente, puntos de análisis para determinar composición química elemental

Tabla 5. Medición de la composición química mediante GDOES en los puntos indicados

\begin{tabular}{ccccccccccc}
\hline \multirow{2}{*}{ Punto } & $\begin{array}{c}\mathbf{F e} \\
{[\mathbf{\%}]}\end{array}$ & $\begin{array}{c}\mathbf{C} \\
{[\mathbf{\%}]}\end{array}$ & $\begin{array}{c}\mathbf{M n} \\
{[\mathbf{\%}]}\end{array}$ & $\begin{array}{c}\mathbf{S i} \\
{[\mathbf{\%}]}\end{array}$ & $\begin{array}{c}\mathbf{P} \\
{[\mathbf{\%}]}\end{array}$ & $\begin{array}{c}\mathbf{S} \\
{[\mathbf{\%}]}\end{array}$ & $\begin{array}{c}\mathbf{N i} \\
{[\mathbf{\%}]}\end{array}$ & $\begin{array}{c}\mathbf{C u} \\
{[\mathbf{\%}]}\end{array}$ & $\begin{array}{c}\mathbf{A l} \\
{[\mathbf{\%}]}\end{array}$ & $\begin{array}{c}\mathbf{Z n} \\
{[\%]}\end{array}$ \\
\hline $\mathbf{1}$ & 0,78 & 0,131 & 5,671 & 0,184 & 0,321 & 0,6831 & 0,153 & 8,973 & $\mathbf{8 1 , 8 9 1}$ & 0,298 \\
$\mathbf{2}$ & 0,78 & 0,133 & 5,588 & 0,188 & 0,3017 & 0,7894 & 0,153 & 8,895 & $\mathbf{8 1 , 9 7 1}$ & 0,294 \\
$\mathbf{3}$ & 0,73 & 0,124 & 5,513 & 0,173 & 0,3363 & 0,8181 & 0,183 & 9,08 & $\mathbf{8 1 , 8 5 1}$ & 0,277 \\
$\mathbf{4}$ & 61,93 & 0,063 & 1,079 & 0,222 & 0,0465 & 0,0148 & 0,128 & 3,228 & 32,786 & 0,094 \\
$\mathbf{5}$ & $\mathbf{9 7 , 8 4}$ & 0,131 & 0,849 & 0,185 & 0,0138 & 0,0102 & 0,185 & 0,504 & 0,019 & 0,007 \\
$\mathbf{6}$ & $\mathbf{9 7 , 8 8}$ & 0,134 & 0,828 & 0,18 & 0,0134 & 0,0097 & 0,181 & 0,494 & 0,018 & 0,009 \\
$\mathbf{7}$ & $\mathbf{9 7 , 8 7}$ & 0,133 & 0,833 & 0,182 & 0,0128 & 0,0097 & 0,181 & 0,505 & 0,013 & 0,006 \\
\hline
\end{tabular}

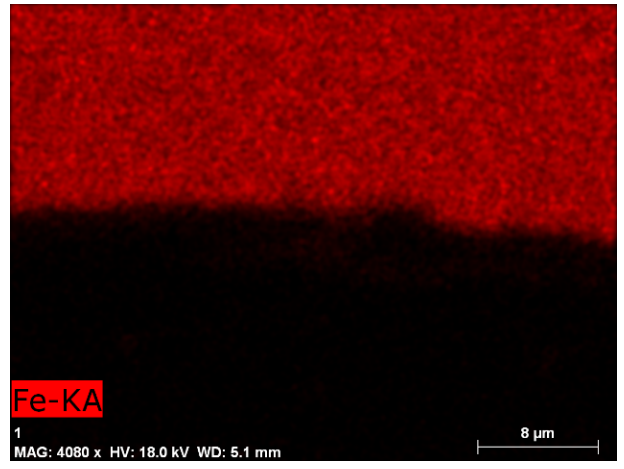

(a)

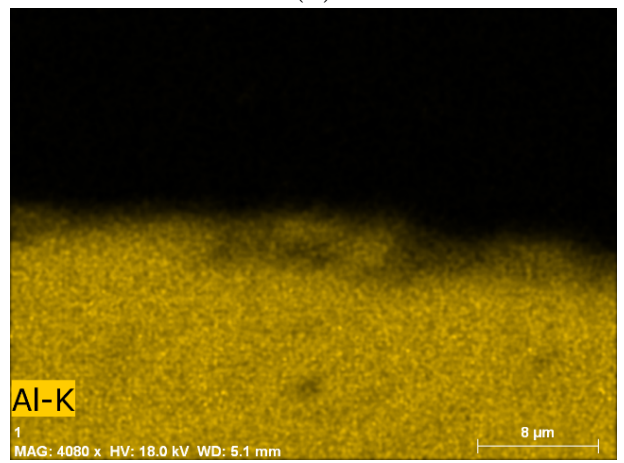

(b)

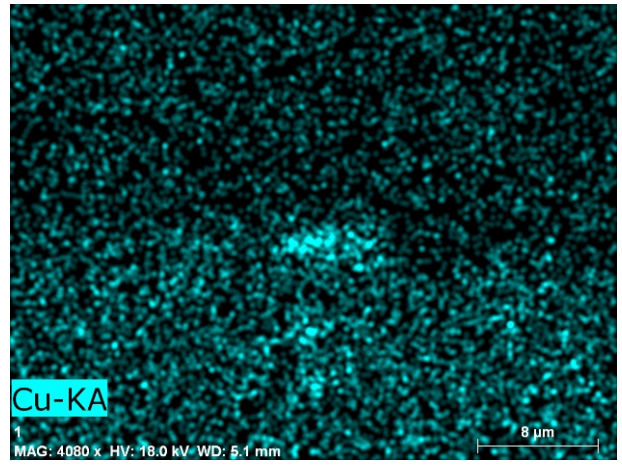

(c)

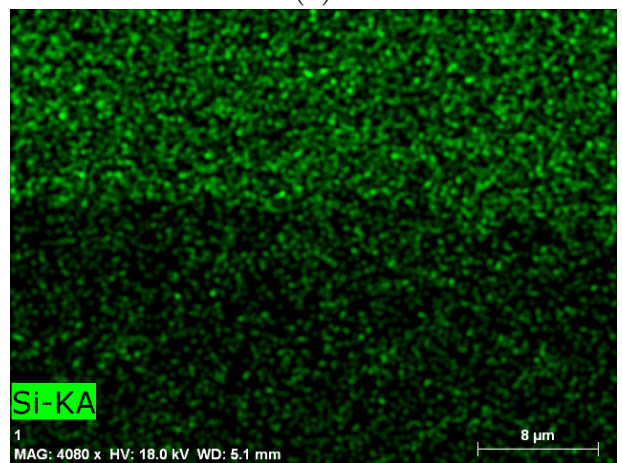

(d)

Figura 10. Espectrometría de emisión óptica de descarga luminiscente, puntos de análisis para determinar composición química elemental 


\section{Conclusiones}

Se desarrolló un sistema de soldadura por fricción con asistencia láser donde se puede controlar la velocidad de rotación del sistema mediante un variador de frecuencia. La velocidad óptima escogida a través de ensayos experimentales corresponde a un rango entre 1600 y $1800 \mathrm{rpm}$.

El sistema de presión hidráulico desarrollado es capaz de proporcionar una fuerza axial sobre los $9800 \mathrm{~N}$, permitiendo realizar soldaduras en ejes de acero-aluminio con diámetros de hasta $20 \mathrm{~mm}$. El rango de presiones para generar fricción y acelerar el proceso de soldadura se encuentra en el rango de 14 a 21 Mpa.

La aplicación del haz láser sobre el eje de acero permite homogenizar la microestructura a la vez que acelera el proceso de soldadura para que exista una mayor interfaz de soldadura entre los ejes de acero y aluminio, mismos que se han verificado en los análisis de la microestructura.

Se determinó el tamaño de grano para cada elemento, para el acero un promedio de grano ASTM 8 con diámetro máximo de 6 micrones. Para el aluminio se tiene un tamaño de grano ASTM 9 y un diámetro máximo de 3 micrones.

En la interfaz de soldadura se determinó un decrecimiento del hierro (Fe) y aluminio ( $\mathrm{Al})$. El Fe disminuye de $97,8 \%$ a $61,9 \%$, mientras que el aluminio se pasa de $81,9 \%$ a $32,8 \%$. También se pudo determinar la existencia de manganeso, cobre y silicio en menor proporción que los materiales base.

\section{Referencias}

[1] A. Handa and V. Chawla, "Experimental evaluation of mechanical properties of friction welded dissimilar steels under varying axial pressures," Strojnícky časopis Journal of Mechanical Engineering, vol. 66, no. 1, pp. 27-36, 2016. [Online]. Available: https://doi.org/10.1515/scjme-2016-0008

[2] S. D. Meshram, T. Mohandas, and G. M. Reddy, "Friction welding of dissimilar pure metals," Journal of Materials Processing Technology, vol. 184, no. 1, pp. 330-337, 2007. [Online]. Available: https: //doi.org/10.1016/j.jmatprotec.2006.11.123

[3] W. Cai, G. Daehn, A. Vivek, J. Li, H. Khan, R. S. Mishra, and M. Komarasamy, "A State-of-the-Art Review on Solid-State Metal Joining," Journal of Manufacturing Science and Engineering, vol. 141, no. 3, p. 031012, 01 2019, 031012. [Online]. Available: https://doi.org/10.1115/1.4041182

[4] P. Sathiya, S. Aravindan, and A. Noorul Haq, "Effect of friction welding parameters on me- chanical and metallurgical properties of ferritic stainless steel," The International Journal of Advanced Manufacturing Technology, vol. 31, no. 11, pp. 1076-1082, Feb. 2007. [Online]. Available: https://doi.org/10.1007/s00170-005-0285-5

[5] A. Hascalik and N. Orhan, "Effect of particle size on the friction welding of $\mathrm{Al}_{2} \mathrm{O}_{3}$ reinforced $6160 \mathrm{Al}$ alloy composite and SAE 1020 steel," Materials and Design, vol. 28, no. 1, pp. 313-317, 2007. [Online]. Available: https://doi.org/10.1016/j.matdes.2005.06.001

[6] N. Ozdemir, F. Sarsilmaz, and A. Hascalik, "Effect of rotational speed on the interface properties of friction-welded AISI 304L to 4340 steel," Materials and Design, vol. 28, no. 1, pp. 301-307, 2007. [Online]. Available: http://dx.doi.org/10.1016/j.matdes.2005.06.011

[7] W.-Y. Li, M. Yu, J. Li, G. Zhang, and S. Wang, "Characterizations of $21-4 \mathrm{~N}$ to $4 \mathrm{Cr}_{9} \mathrm{Si}_{2}$ stainless steel dissimilar joint bonded by electric-resistance-heat-aided friction welding," Materials and Design, vol. 30, no. 10, pp. 4230-4235, 2009. [Online]. Available: https://doi.org/10.1016/j.matdes.2009.04.032

[8] E. Taban, J. E. Gould, and J. C. Lippold, "Dissimilar friction welding of 6061T6 aluminum and AISI 1018 steel: Properties and microstructural characterization," Materials and Design (1980-2015), vol. 31, no. 5, pp. 2305-2311, 2010. [Online]. Available: https://doi.org/10.1016/j.matdes.2009.12.010

[9] S. Fukumoto, H. Tsubakino, K. Okita, M. Aritoshi, and T. Tomita, "Amorphization by friction welding between 5052 aluminum alloy and 304 stainless steel," Scripta Materialia, vol. 42, no. 8, pp. 807-812, 2000. [Online]. Available: https://doi.org/10.1016/S1359-6462(00)00299-2

[10] A. Handa and V. Chawla, "Experimental study of mechanical properties of friction welded AISI 1021 steels," Sadhana, vol. 38, no. 6, pp. 1407-1419, Dec. 2013. [Online]. Available: https://doi.org/10.1007/s12046-013-0181-x

[11] J. Luo, Y. H. Ye, J. J. Xu, J. Y. Luo, S. M. Chen, X. C. Wang, and K. W. Liu, "A new mixed-integrated approach to control welded flashes forming process of damping-tube-gland in continuous drive friction welding," Materials and Design, vol. 30, no. 2, pp. 353-358, 2009. [Online]. Available: https://doi.org/10.1016/j.matdes.2008.04.075

[12] S. Celik and I. Ersozlu, "Investigation of the mechanical properties and microstructure of 
friction welded joints between AISI 4140 and AISI 1050 steels," Materials \& Design, vol. 30, no. 4, pp. 970-976, 2009. [Online]. Available: https://doi.org/10.1016/j.matdes.2008.06.070

[13] J. L. Mullo, J. A. Ramos-Grez, and G. O. Barrionuevo, "Effect of laser heat treatment on the mechanical performance and microstructural evolution of AISI 1045 steel2017-T4 aluminum alloy joints during rotary friction welding," Journal of Materials Engineering and Performance, vol. 30, no. 4, pp. 2617-2631, Apr. 2021. [Online]. Available: https://doi.org/10.1007/s11665-021-05614-6

[14] G. L. Wang, J. L. Li, W. L. Wang, J. T. Xiong, and F. S. Zhang, "Rotary friction welding on dissimilar metals of aluminum and brass by using pre-heating method," The International Journal of Advanced Manufacturing Technology, vol. 99, no. 5, pp. 1293-1300, Nov. 2018. [Online]. Available: https://doi.org/10.1007/s00170-018-2572-y

[15] S. Celik and I. Ersozlu, "Investigation of the mechanical properties and microstructure of friction welded joints between AISI 4140 and AISI 1050 steels," Materials and Design, vol. 30, no. 4, pp. 970-976, 2009. [Online]. Available: https://doi.org/10.1016/j.matdes.2008.06.070

[16] W. Li, A. Vairis, M. Preuss, and T. Ma, "Linear and rotary friction welding review," International Materials Reviews, vol. 61, no. 2, pp. 71-100, 2016. [Online]. Available: https://doi.org/10.1080/09506608.2015.1109214

[17] P. Ferro, Y. Wei, and F. Sun, "Microstructures and mechanical properties of $\mathrm{Al} / \mathrm{Fe}$ and $\mathrm{Cu} / \mathrm{Fe}$ joints by continuous drive friction welding," $\mathrm{Ad}$ vances in Materials Science and Engineering, vol. 2018, p. 2809356, Jun. 2018. [Online]. Available: https://doi.org/10.1155/2018/2809356

[18] O. D. Hincapié, J. A. Salazar, J. J. Restrepo, J. A. Graciano-Uribe, and E. A. Torres, "Weldability of aluminum-steel joints using continuous drive friction welding process, without the presence of intermetallic compounds," Engineering Journal, vol. 24 , no. 1, pp. 129-144, 2020. [Online]. Available: https://doi.org/10.4186/ej.2020.24.1.129

[19] X. Li, J. Li, F. Jin, J. Xiong, and F. Zhang, "Effect of rotation speed on friction behavior of rotary friction welding of aa6061-t6 aluminum alloy," Welding in the World, vol. 62, no. 5, pp. 923-930, Sep. 2018. [Online]. Available: https://doi.org/10.1007/s40194-018-0601-y
[20] E. Taban, J. E. Gould, and J. C. Lippold, "Dissimilar friction welding of 6061T6 aluminum and AISI 1018 steel: Properties and microstructural characterization," Materials and Design (1980-2015), vol. 31, no. 5, pp. 2305-2311, 2010. [Online]. Available: https://doi.org/10.1016/j.matdes.2009.12.010

[21] X. Fei, X. Jin, N. Peng, Y. Ye, S. Wu, and H. Dai, "Effects of filling material and laser power on the formation of intermetallic compounds during laser-assisted friction stir butt welding of steel and aluminum alloys," Applied Physics A, vol. 122, no. 11, p. 936, Oct. 2016. [Online]. Available: https://doi.org/10.1007/s00339-016-0462-4

[22] T. Wada, Y. Morisada, Y. Sun, H. Fujii, Y. Kawahito, M. Matsushita, and R. Ikeda, "Friction stir welding of medium carbon steel with laser-preheating," ISIJ International, vol. 60, no. 1, pp. 153-159, 2020. [Online]. Available: https://doi.org/10.2355/isijinternational. ISIJINT-2019-394

[23] X. Fei, J. Li, W. Yao, and L. Jin, "Study of temperature on microstructure and mechanical properties on $\mathrm{Fe} / \mathrm{Al}$ joint in laser-assisted friction stir welding," AIP Advances, vol. 8, no. 7, p. 075214, 2018. [Online]. Available: https://doi.org/10.1063/1.5039417

[24] M. Kutsuna, N. Yamagami, M. J. Rathod, and H. Y. A. Ammar, "Laser roll welding for joining of low-carbon steels to aluminium alloys," Welding International, vol. 20, no. 6, pp. 446-456, 2006. [Online]. Available: https://doi.org/10.1533/wint.2006.3599

[25] Q. Guan, J. Long, P. Yu, S. Jiang, W. Huang, and J. Zhou, "Effect of steel to aluminum laser welding parameters on mechanical properties of weld beads," Optics and Laser Technology, vol. 111, pp. 387-394, 2019. [Online]. Available: https://doi.org/10.1016/j.optlastec.2018.09.060

[26] S. L. Campanelli, G. Casalino, C. Casavola, and V. Moramarco, "Analysis and comparison of friction stir welding and laser assisted friction stir welding of aluminum alloy," Materials (Basel, Switzerland), vol. 6, pp. 5923-5941, Dec 2013. [Online]. Available: https://doi.org/10.3390/ma6125923

[27] M. Merklein and A. Giera, "Laser assisted friction stir welding of drawable steelaluminium tailored hybrids," International Journal of Material Forming, vol. 1, no. 1, pp. 1299-1302, Apr. 2008. [Online]. Available: https://doi.org/10.1007/s12289-008-0141-x 
[28] M. Yilmaz, M. Çöl, and M. Acet, "Interface properties of aluminum/steel friction-welded components," Materials Characterization, vol. 49, no. 5, pp. 421-429, 2002. [Online]. Available: https://doi.org/10.1016/S1044-5803(03)00051-2
[29] N. Hynes, P. Nagaraj, and J. Sujana, "Investigation on joining of aluminum and mild steel by friction stud welding," Materials and Manufacturing Processes, vol. 27, no. 12 , pp. 1409-1413, 2012. [Online]. Available: https://doi.org/10.1080/10426914.2012.667894 